\title{
A novel LBP-Mean shift segmentation algorithm for UAV remote sensing images based on LBP textural features and improved Mean shift algorithm
}

\author{
Surong Xiang ${ }^{1, a}$, Jingwen $\mathrm{Xu}^{1, \mathrm{~b}^{*}}$, Junfang $\mathrm{ZHAO}^{2 \mathrm{c}}$,Yong $\mathrm{Li}^{1, \mathrm{~d}}$ and Shaoyao \\ ${ }^{1}$ College of Environment, Sichuan Agricultural University, Chengdu 611130, China \\ ${ }^{2}$ Chinese Academy of Meteorological Sciences, CMA, Beijing 100081, P.R. China \\ aemail:xiangxiang63@126.com, *bCorresponding author: x.j.w@163.com,
}

Keywords: Computer vision; Hyperspectral remote-sensing images; image segmentation; mean shift; LBP texture feature

Abstract. The proposed method deals with the joint use of the textural features and the image edge by the remote-sensing images.A definition of an adaptive segmentation algorithm is considered.Based on LBP features detecting,the textural information associated with each cell images is extracted as the set of connected cell with an similar flag value to which the cell belongs: The cell's neighborhood is characterized by the color space and light intensity distribution of the corresponding flag zone.The textural information is the original cell's value, be it a distinctive characteristic.Using Mean shift algorithm, the edge information are jointly used for the segmentation through a change of distance formula. Experiments on hyperspectral and panchromatic images are presented and show a significant increase in segmentation accuracies for village area:For instance, with the overall accuracy is increased from smash with a conventional algorithm to integrity with the proposed approach. Comparisons with other related software methods show that the method is competitive.

\section{Introduction}

With recent advancing in remote sensor technology, the segmentation of high resolution remote sensing images has become a challenging problem. Spatial resolution is now as high as $0.75 \mathrm{~m}$ for several satellites, e.g. IKONOS, QUICKBIRD ${ }^{[1]}$. Meanwhile,the meter and sub-meter resolution remote sensing images, represented by SPOT5, Quick Bird, and IKONOS have been widely used in various fields. New satellite sensors acquire images with high spectral and spatial resolution, and the revisiting time is constantly reduced ${ }^{[2]}$. As a consequence, with such resolution many small objects and materials can now be extracted with very fine accuracy for detection, classification or segmentation. Many of the applications remain to be explored and specific methodologies need to be developed to handle the complex properties of very high resolution images ${ }^{[1]}$.

It is conventional to use spectral information as an input to the classifier for the critical problem of high resolution remote sensing images.Automation has been a hot issue in constructions extraction, but there has not yet been a universally accepted algorithm ${ }^{[3]}$.

\section{LBP operator and Mean Shift algorithm}

Texture analysis is through a certain image processing techniques to extract texture features, to obtain texture quantitative or qualitative description of the process. At the university of Oulu, Finland (Oulu) T.O jala50 et al in 1996 put forward by using local Binary pattern (Loca1 Binary Patterns, LBP) operator is used to analyze the image texture features ${ }^{[2]}$. Studies have shown that LBP operator is a kind of effective texture description operator, it has a rotating invariance and gray scale in variance and other significant advantages.

Mean Shift algorithm is first applied in medical image and the study of natural images, the algorithm is efficient and robust, do not need prior knowledge, we try to bring it into remote sensing image segmentation. Yong Li etal. have proposed an algorithm which combines adaptive mean shift 
with LBP and Canny Features ${ }^{[4]}$.Implying LBP and Canny Features into mean shift, it Combined with a variety of characteristics to detect optimal clustering number of mean shift., which better fits human visual perception.

\section{Data sets}

A shot of Meishan city, Sichuan province of China Danleng county UAV image are selected as the segmentation experiment data to evaluate the effectiveness of the proposed approach. UAV has characteristics that the light weight wind resistance is poor; to obtain the projection distortion of big and small images like picture; unstable flight attitude makes for the image of the apparent overlap degree of deviation so that can affect image orthographic processing ${ }^{[5]}$.

\section{Operation platform}

This experiment platform for the frequency of 2.1 GHZ Intel CORE2 duo processor, winXP / 7 operating systems, 2 GB of memory, visual studio 2010 as implementation platform, $\mathrm{c}++$ is the programming language.

\section{Process and Method}

\section{Text and indenting}

Local binary pattern

Local Binary Pattern (Local Binary Pattern, LBP) ${ }^{[5]}$ by T.o jala, etc in 1996 , because of its simple theory, calculation, are widely used in image processing field.The limitation of the basic LBP operator is its small $3 * 3$ neigh-borhood which can not capture dominant features with large scale structures. Hence the operator later was extended to use neighbor-hood of different sizes. Then convenient for calculation and expression we will often binary code is converted to a decimal number as the final eigenvalue for subsequent calculation:

(4-1)

There into $P=8, R=1$.

$$
L B P_{P, R}=\sum_{i=0}^{P-1} 2^{i} s\left(g_{c}, g_{i}\right) \quad \begin{aligned}
& \text { 4.1.2Facial UAV images using } L B P \\
& \text { Firstly,converts remote sensing imag }
\end{aligned}
$$

Firstly,converts remote sensing image to a greyscale image,because the LBP is analysis of image gray level information processing.then the image is calculated according to the template LBP operator, and get each pixels, the new value, then the output LBP code value. As shown in figure (as shown in Fig. 1) is several kinds of LBP operator LBP code diagram of the remote sensing image.

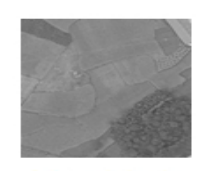

The original gray scale

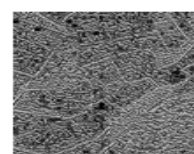

elementary

LBP $₫=8, R=1.0$ )

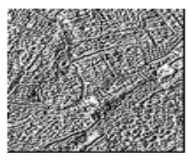

multiscale

LBP $(\mathrm{P}=8, \mathrm{R}=2.0$ )
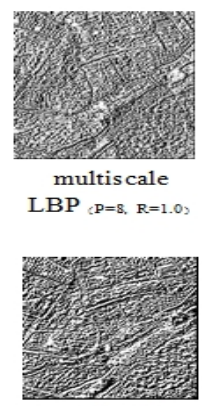

multiscale

LBP $_{C P=16, R=2.0}$

Fig. 1 UVA images Several kinds of LBP code diagram of the remote sensing image 


\section{The proposed LBP- Meanshift segmentation approach}

In this section, the LBP- Mean shift algorithm segmentation considered approach is definited. The aim of this strategy is to find the best segmentation result from a Multi-scale complex algorithm by joining LBP feature textural to Mean shift algorithm to the user.

\section{Using an improved mean shift algorithm}

The MS algorithm is a non-parametric, iterative technique for locating modes of a pdf obtained via a kernel density estimate from a given data $\operatorname{set}^{[20]}$. These modes play an important role in many machine learning applications, such as classification, image segmentation, and object tracking ${ }^{[20]}$. Mean shift algorithm for image segmentation of the specific process:(1)calculate the image characteristics of the five dimensions of space $(x, y, l, u, v)$.(2)Setting (space bandwidth $h s$, $Y h s X h s-1,1)$ the color of the characteristic values for $(L, U, V)$, within the bandwidth of each pixel point $(X h s-I, Y h s-j(I, j=0,1$. The $h s-2))(L, U, V)$ of the value of the di, j for pixels in bandwidth

$$
d_{i, j}=\sqrt{(L-l)^{2}+(U-u)^{2}+(V-v)^{2}} \quad \text { average distance formula }(2-1) .
$$

MS is used to find the peak of the distance value $\mathrm{d}(y)$, which is referred to literature ${ }^{[4]}$.

\section{Boosting LBP for mean shift algorithm}

Traditional Mean shift algorithm for image segmentation, will only like space position and color information of yuan as a feature vector, which improve the precision of image segmentation and boundary is not clear enough, and low efficiency.In this paper, based on LBP texture feature vector and improved Mean shift algorithm of LBP - Mean shift algorithm for high resolution image segmentation.Algorithm specific process is as follows:(1)By using multi-scale LBP algorithm to calculate the high resolution image of LBP values.(2)Use first (formula (4-2) improve the traditional Mean shift algorithm of calculating the distance between the color formula, the image to get rid of

$$
\begin{aligned}
& d l_{(i, j)}=|L-l| ; \\
& d u_{(i, j)}=|U-u| \\
& d v_{(i, j)}=|V-v| ; \\
& D_{(i, j)}=\max \left(d l_{(i, j)}, d u_{(i, j)}, d v_{(i, j)}\right)
\end{aligned}
$$
the influence of some "salt and pepper noise" (bandwidth setting smaller space, color larger bandwidth Settings.).

(3)On the basis of the improved Mean shift algorithm to make certain improvement. Add a LBP $D b_{(i, j)}=|B-b| \begin{aligned} & \text { texture feature in the feature vector, in the process of calculation in the } \\ & \text { color feature vector channel add a LBP values, namely }(x, y, l, u, v, b) b\end{aligned}$ for LBP eigenvalues. LBP feature distance formula formula (4-4), add a LBP values bandwidth parameter hl.

\section{(4-4)}

\section{LBP-Meanshit algorithm segmentation}

Steps of calculation:

(1)to calculate LBP graph:LBP algorithm is used to calculate the remote sensing image LBP figure .

(2)The Mean shift initial smoothing of uav remote sensing image.In order to get rid of some "salt and pepper noise" on the result of remote sensing image segmentation, first using the improved Mean 
shift algorithm for image processing, spatial location bandwidth is set to 1 , the bandwidth is set to 255 colors.(3)to join the LBP feature value iterative calculation scheme shift + LBP figure.

In traditional Mean shift algorithm add LBP texture characteristic value of channel, participate in the division of Mean shift algorithm calculation, the result diagram and calculate the new LBP. And then participate in the improved Mean shift method, this method of iteration, the number of iterations to 10 (image 1 ), the spatial bandwidth set to 4 , color bandwidth is set to 8 , LBP values bandwidth initial values for 10, 20 each iteration. The result shown in Fig.2.line space).

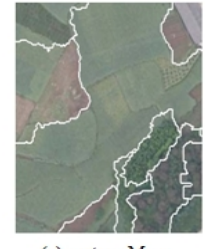

(a) custom Mean

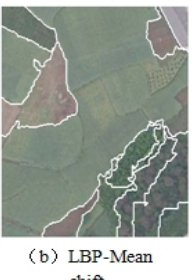

shift

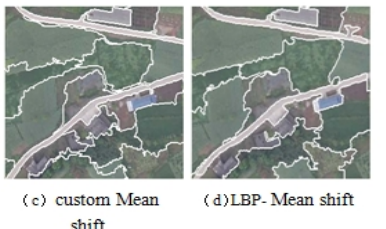

shift

Fig.2UAV remote sensing images of two kinds of Mean shift segmentation region merging processing result contrast figure.

\section{Comparison}

With EDISON and eCognition software comparing with LBP - meanshift operator segmentation results. In conclusion, this paper presents the results of LBP-Meanshift algorithm segmentation than EDISON and eCognition software on the accuracy of the results of segmentation have obvious enhancement, segmentation result more accord with human visual perception.
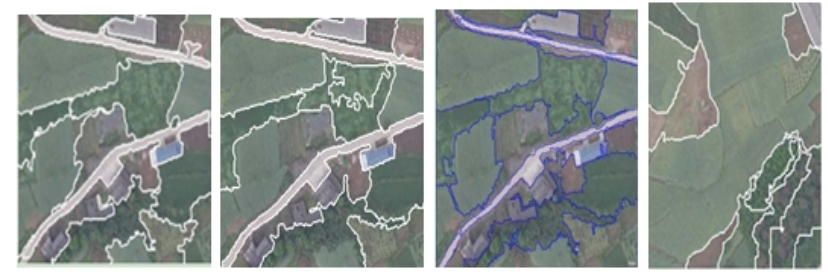

(a) custom Mean shift (b) LBP-Mean shift (c) EDISON software (d) eCognition software

Fig. 3 UVA remote sensing image of the improved Mean shift and EDISON software and eCongnition results contrast figure

\section{Conclusions}

Paper's main research conclusion is as follows:

(1)This paper join LBP texture characteristic value in the improved Mean shift segmentation algorithm can realize the automatic remote sensing image segmentation, segmentation accuracy is higher, the effect more accord with human visual perception.

(2)Comparing the results of segmentation of labeled edge of the area with the traditional Mean shift merge segmentation results and EDISON and eCognition.Visible, LBP-Meanshift algorithm is superior to the traditional Mean shift method and EDISON software integral effect, to a certain extent, better than software eCognition segmentation effect.

\section{Acknowledgements}

This study is financially supported by National Natural Science Foundation of China (31101073), the Natural Scientific Research Fund of the Education Department of Sichuan Province (09ZA075), Open Research Fund Program of the Meteorological Center for Huaihe watershed (HRM200905). 


\section{References:}

[1] M. Fauvel, J. Chanussot, J.A. Benediktsson, A spatial-spectral kernel-based approach for the classification of remote-sensing images, Pattern Recognition 45 (2012) 381-392.

[2] D. Comaniciu, P. Meer, Mean shift analysis and applications, in: Proceedings ofthe Seventh IEEE International Conference on Computer Vision, vol. 2, 1999,pp. 1197-1203.

[3] Ojala, T.,Pietikainen, M.,Maenpaa, T. Multiresolution gray-scale and rotation invariant texture classification with local binary patterns[J]. Pattern Analysis and Machine Intelligence, IEEE Transactions on, 2002, 24 (7): 971-987.

[4]Yong Li, Jingwen Xu, Junfang ZHAO, Yudan ZHAOland Xin Li,An Improved Mean shift Segmentation Method of High-resolution Remote Sensing Image Based on LBP and Canny Features,Applied Mechanics and Materials Vols.

[5]Y. Guo, H.D. Cheng, J. Tian, Y. Zhang, A novel approach to speckle reduction inultrasound imaging, Ultrasound Med. Biol. 35 (2009) 628-640. 